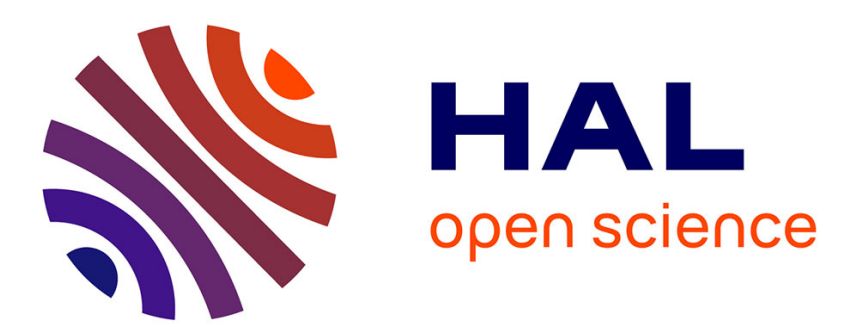

\title{
Identification of adhesive properties in GLARE assemblies using Digital Image Correlation
}

Roberto Fedele, Bumedijen Raka, François Hild, Stéphane Roux

\section{To cite this version:}

Roberto Fedele, Bumedijen Raka, François Hild, Stéphane Roux. Identification of adhesive properties in GLARE assemblies using Digital Image Correlation. Journal of the Mechanics and Physics of Solids, 2009, 57, pp.1003-1016. hal-00376485

\section{HAL Id: hal-00376485 \\ https://hal.science/hal-00376485}

Submitted on 17 Apr 2009

HAL is a multi-disciplinary open access archive for the deposit and dissemination of scientific research documents, whether they are published or not. The documents may come from teaching and research institutions in France or abroad, or from public or private research centers.
L'archive ouverte pluridisciplinaire HAL, est destinée au dépôt et à la diffusion de documents scientifiques de niveau recherche, publiés ou non, émanant des établissements d'enseignement et de recherche français ou étrangers, des laboratoires publics ou privés. 


\title{
Identification of adhesive properties in GLARE assemblies using Digital Image Correlation
}

\author{
R. Fedele, ${ }^{* \dagger}$ B. Raka,${ }^{\ddagger}$ F. Hild, ${ }^{\ddagger}$ and S. Roux ${ }^{\ddagger}$ \\ ${ }^{\dagger}$ Department of Structural Engineering, Politecnico di Milano \\ piazza Leonardo da Vinci 32, 20133, Milan, Italy. \\ ${ }^{\ddagger}$ LMT Cachan, ENS Cachan / CNRS / UPMC / PRES UniverSud Paris \\ 61 avenue du Président Wilson, F-94235 Cachan Cedex, France.
}

\begin{abstract}
An experimental-numerical methodology is introduced to identify the parameters of a cohesive law of an adhesive layer within a joined assembly on the basis of kinematic data provided by digital image correlation. Nonconventional experiments on joined samples were designed to generate within the assembly and the adhesive film complex strain and stress states close to those expected in service and up to complete debonding. The modeling is developed with reference to the observed sub-domain in which the experimental boundary conditions are prescribed. The non-linear behavior of the adhesive layer is described as a finite thickness interface endowed with a mixed-mode cohesive law whose parameters are identified so as to match at best the measured displacement field.
\end{abstract}

Key words: Cohesive crack model; Digital Image Correlation; Inverse Problems; Parameter identification; Structural adhesives.

\footnotetext{
*Corresponding author. Fax: +39 022399 4220. E-mail: fedele@stru.polimi.it
} 


\section{Introduction}

Joining two materials to obtain a better performance as that of the singles is a general goal in composite design. Various architectures are obtained when dealing with layers or fibers. Among these, GLARE has received some attention in the aerospace industry (Vlot, 2001; Sinke, 2003). GLARE is a "GLAss-REinforced" fiber metal laminate, composed of several layers of aluminum alloy interspersed with layers of glass-fiber "pre-preg." In the design of stiffened panels for aircraft structures, recourse has also been made to adhesively-bonded assemblies between GLARE laminates with different geometries. To predict the structural response of such assemblies, a good understanding of the adhesive layer properties is required. In this paper, it is proposed to use images of the joined assembly in a non-conventional mechanical test, representative of in-service conditions, to identify the parameters governing a mixed-mode cohesive model for the adhesive layer.

When dealing with model calibration of adhesive joints, point data, e.g., strain and load data, are usually the only experimental information available (Derewonko et al., 2008). Pictures at different scales are used in a qualitative way in addition to global data (Pardoen et al., 2005; Leffler et al., 2007; Sørensen et al., 2008; Salomonsson and Andersson, 2008), or quantitatively by evaluating deflections (Yang et al., 2001; Su et al., 2004) and deformed shapes (Sargent, 2005). In this study, it is proposed to use full-field measurements provided by Digital Image Correlation (DIC). Its advantage lies in the fact that numerous measurement points are available to analyze 
an experiment, as opposed to the standard practice of using few data.

During the last years, DIC (Sutton et al., 2000) has undergone substantial developments for several reasons. First, its application is generally simple and straightforward. It works effectively under natural light. The resolution is of the same order as many conventional measurement devices. It is employable at different scales, from macro down to micro or even nano scales (Sutton et al., 1999; Soppa et al., 2001; Chasiotis and Knauss, 2002; Forquin et al., 2004; Chasiotis, 2007). In all the previous cases, DIC was based upon local matching of interrogation windows in a series of pictures. An alternative approach, based on a finite-element discretization of the displacement field (Besnard et al., 2006), will be used herein.

Several attempts have been made to enrich the experimental information gathered by a conventional equipment with full-field measurements (McNeill et al., 1987; Geers et al., 1999; Abanto-Bueno and Lambros, 2002; Cho et al., 2005; Maier et al., 2005; Hild and Roux, 2006). However, very few studies deal with the identification of cohesive models because of experimental challenges and the scales at which data are to be obtained. Abanto-Bueno and Lambros (2005) used a multi-camera system to determine the traction separation law. The near-field images were used to measure the opening displacements, and the far-field views to evaluate the interface tractions via an elastic solution. The above procedure, however, is not a reliable route to follow when the adherend behavior is nonlinear and only microscopic measurements are used (as in the present case).

Advanced mechanical models for describing the nonlinear behavior of materials and structures contain numerous parameters, many of which are not 
easy to identify. Therefore, there is strong interest in industrial environments to develop procedures for estimating these parameters on the basis of innovative experimental tests (Yoshida et al., 2003). The latters are designed with the aim of generating stress and strain states close to those expected in-service. This approach leads to difficult challenges both in terms of measurement and identification. On the measurement side, stress and strains are typically very heterogeneous, possibly at the microscopic scale, or difficult to access. For identification, the usual occurrence of different non-linearities requires a full modeling that often makes the procedures quite difficult. The novel developments, which open the way to such delicate task, is the original combination of experimental full field measurement and inverse problem approaches.

The above (inverse) procedure is applied to the characterization of a structural adhesive layer, joining a laminate skin and a Z-shaped reinforcement (stringer), both made of GLARE for aerospace applications. The experiments, specially designed for the present investigation, were monitored by a digital camera, empowered by a long distance microscope (Section 2). A meaningful sample of pictures was analyzed by a finite element based DIC algorithm. The model adopted herein to describe the response of the monitored Region-of-Interest (ROI) is presented in Section 3. The mechanical properties of the adhesive layer, modeled as a cohesive thick interface law, is estimated via an inverse procedure, and the traction profiles along the adhesive layer reconstructed (Section 4).

Notations. Small strain and displacements are assumed. The symbol : denotes the contraction with respect to two indices, namely, $\boldsymbol{A}: \boldsymbol{B}=A_{i j} B_{i j}$. 
Bold symbols denote vectors, matrices, or tensors, depending on the context.

\section{Debonding experiments}

Non-conventional tests are designed to generate complex stress and strain states within a joined assembly. Under these conditions, nothing impedes distinct non-linear mechanical phenomena from occurring during the test, and interact with each other (e.g., fracture of the adhesive layer, simultaneous plastic yielding of the adherends, or local delamination). Therefore, numerical simulations of the overall specimen response should take into account all these phenomena simultaneously. In this context, displacement measurements, say by DIC, turn out to be advantageous when compared with traditional "point" and overall measurements. In particular, DIC allows for the evaluation of the actual kinematic boundary conditions of the modeled domain. The experiments reported herein are applied to the characterization of a structural (60- $\mu \mathrm{m}$ thick) adhesive layer, joining a (1.4-mm thick) laminate skin and a Z-shaped reinforcement (stringer), both made of GLARE (Figure 1). The single epoxy adhesive layer, shown in Figure 2, is made of BMS 5-101 produced by 3M (commercial denomination AF163 degree 10). Tests were designed and performed on rectangular joined specimens (surface area: $297 \times 50 \mathrm{~mm}^{2}$ ) to lead to complete debonding of the adhesive (Figure 3). The experiments were monitored by a digital camera equipped with a long distance microscope. A small region on the specimen surface around the adhesive layer (of area approximately equal to $2 \times 2.5 \mathrm{~mm}^{2}$ ) was selected as the ROI and monitored during the tests. The pictures at the microscale (Figure 4) were subsequently processed to determine the displacements ex- 


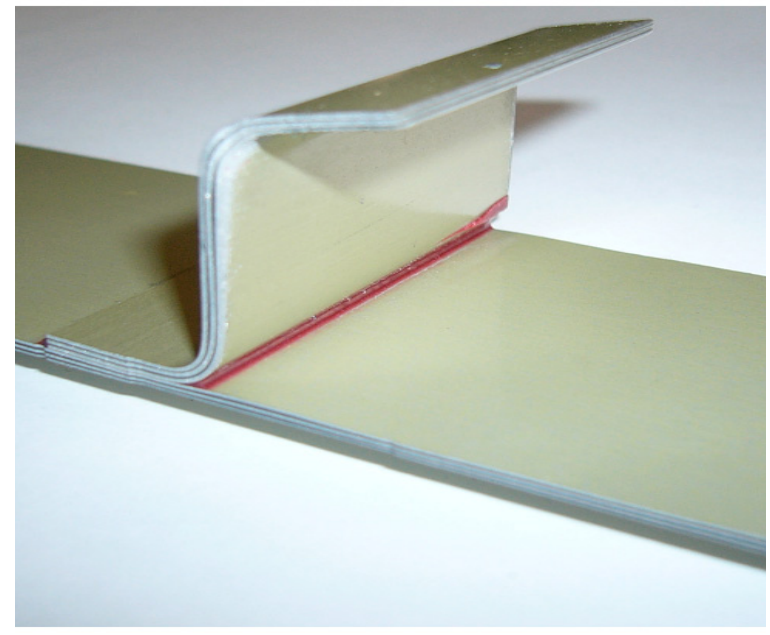

Figure 1: Joined sample (surface area: $297 \times 50 \mathrm{~mm}^{2}$ ) cut from the original laminate. The epoxy adhesive layer between the plate and the Z-shaped stringer is the focus of the present study.

perienced within the ROI by DIC.

The code used herein is based on a Galerkin, finite-element discretization of the displacement field with bilinear shape functions (Besnard et al., 2006). It allows one to establish a close connection with subsequent finite element simulations. Figure 5 shows the kinematic measurements at the microscale provided by DIC, which concern two strips belonging to the joined substrates close to the adhesive layer. The deformed meshes are shown at different instants $i$ during the debonding test. Measurements do not consider the interior part of the adhesive joint, which has a poor texture quality and a vanishing intensity gradient (it appears almost completely dark in the 


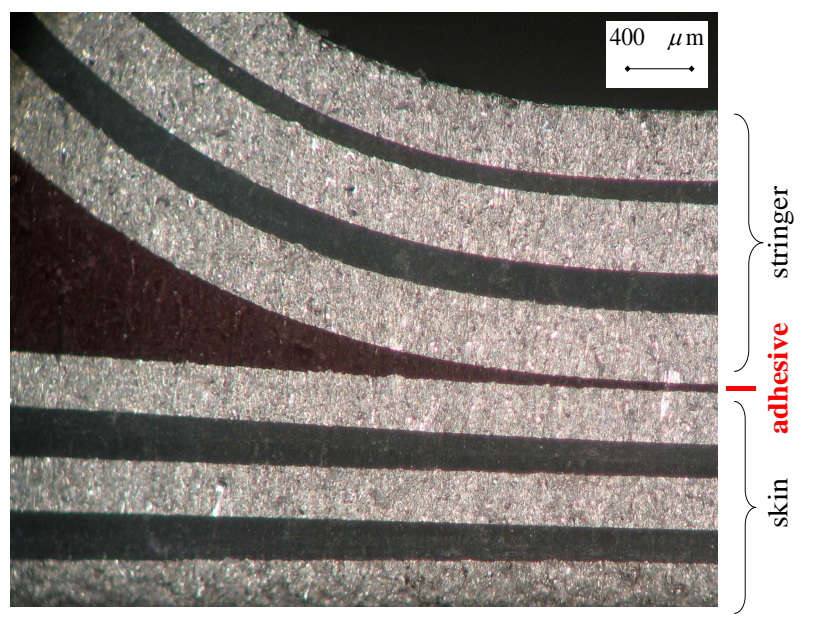

Figure 2: Detail of the adhesive joint (in red), showing a tapered profile to allow for the insertion of the Z-shaped reinforcement on the skin laminate. Both laminates are made of three aluminum alloy (light gray) and two epoxy pre-preg (dark gray) layers. The small sub-domain monitored by DIC is located at the right end of the picture, where the joint thickness stabilizes to $60 \mu \mathrm{m}$ (constant along the remaining length). The magnification used herein is less than that adopted in the pictures analyzed with DIC.

pictures of Figure 4). The adopted finite element (mechanical) model to be introduced in the sequel is consistent with such measurements.

\section{Mechanical model}

The chosen mechanical modeling is matched to the same mechanical problem as in the experiments with the exclusive reference to the considered ROI. Displacement fields measured by means of a DIC procedure are suitable to specify Dirichlet boundary conditions for such a region. The debonding pro- 

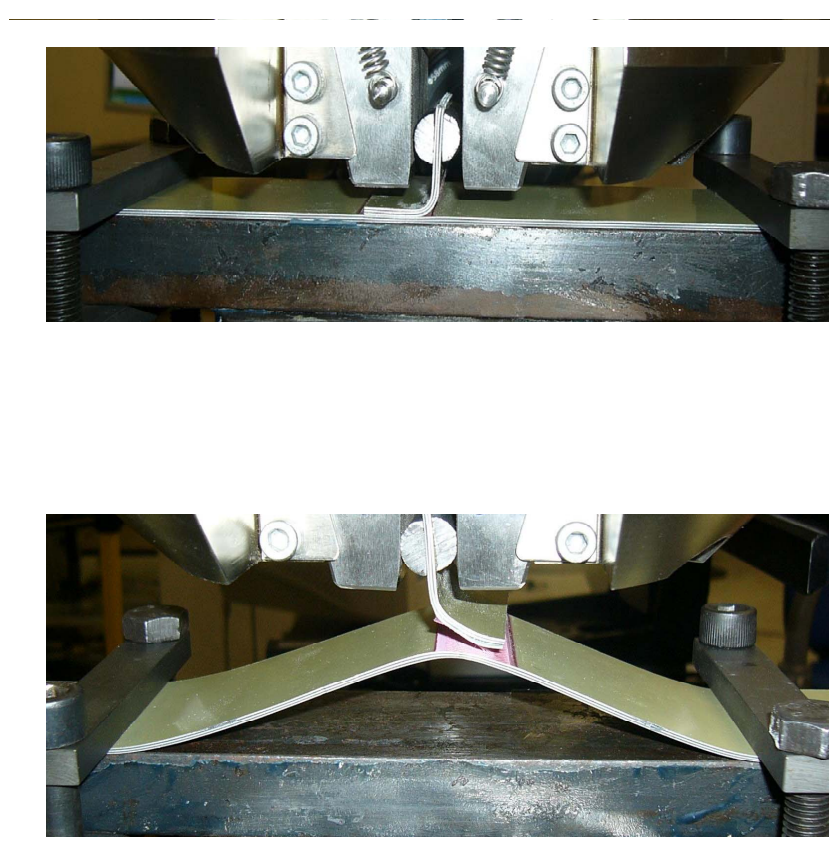

Figure 3: Non-conventional test on a joined sample, up to complete debonding.

cess of the adhesive layer is simulated by prescribing displacements estimated by DIC along the boundary of the monitored sub-domain. The mechanical problem is solved with reference to this sub-domain, with a significant gain in model complexity and computing time. In the mechanical modeling of the debonding process developed herein, the hypothesis of holonomy is assumed for both the adhesive response and the substrate elasto-plastic behavior, namely they are governed by closed-form, history-independent functions accounting for the current values of strains and stresses. It is motivated, as in the deformation theory of plasticity, by the progressive propagation 
0

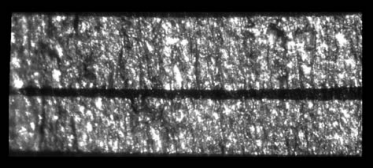

7

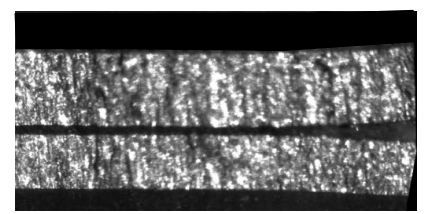

11

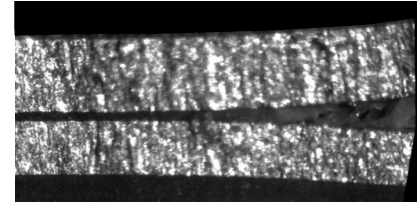

15

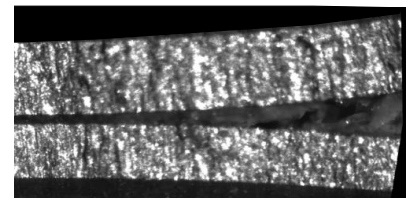

17

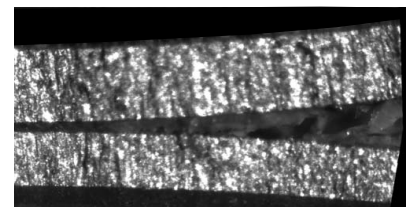

19

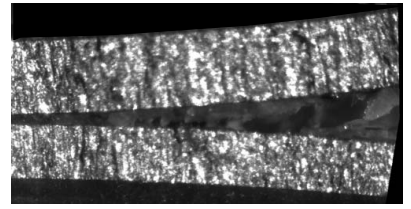

Figure 4: Sequence of digital images monitoring the debonding process of the adhesive layer (about $60 \mu \mathrm{m}$ thick) within the ROI after removal of rigid-body motions. The joint length shown in picture labeled 0 is $1.88 \mathrm{~mm}$. The (glossy) aluminum alloy layers fastened by the (dark) adhesive layer can be clearly distinguished, and the appearance of a completely traction-free surface is preceded by isolated thin fibrils bridging the opposite aluminum alloy surfaces, illuminated by light traversing the open microscopic crack.

of the debonding process. It represents an important simplification from a numerical standpoint, both in the forward simulations and in the analytical sensitivity analysis. 

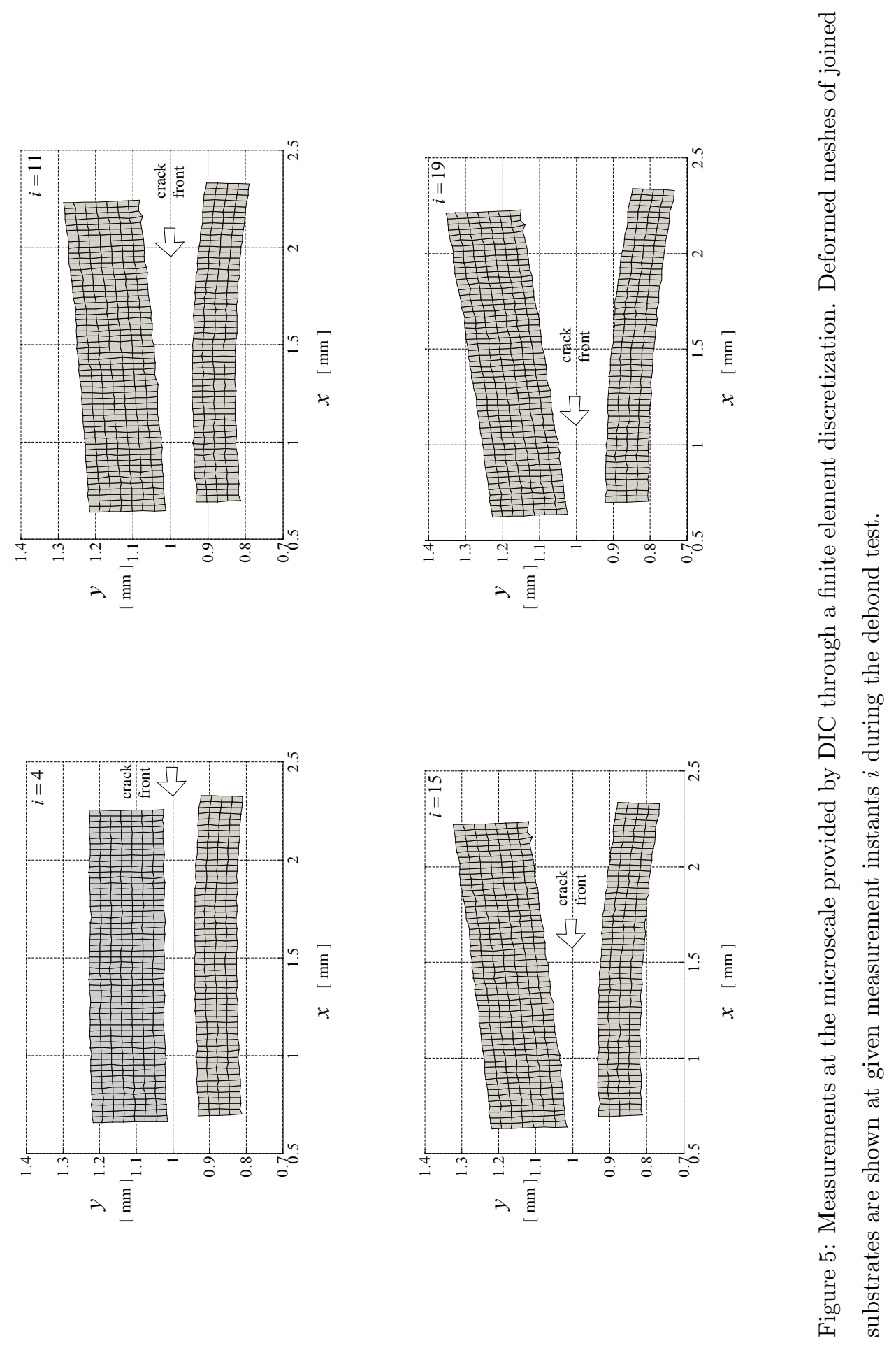

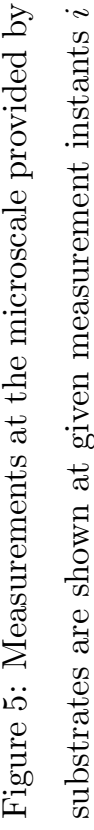




\subsection{Aluminum alloy substrate}

The aluminum (cold-rolled 2024-T3) alloy substrates are modeled according to Prandtl-Reuss' flow rule (Lemaitre and Chaboche, 1990)

$$
\boldsymbol{\epsilon}=\frac{1+\nu}{E} \boldsymbol{S}+\frac{1-2 \nu}{E} p \boldsymbol{I}+\frac{3}{2} \alpha\left(\frac{q}{\sigma_{0}}\right)^{n-1} \boldsymbol{S}
$$

where $\boldsymbol{I}$ denotes the second-order identity tensor, $\boldsymbol{\epsilon}$ the (infinitesimal) strain

tensor, $\boldsymbol{S}$ the stress deviator tensor, $q=\sqrt{\frac{3}{2} \boldsymbol{S}: \boldsymbol{S}}$ its normalized secondorder invariant (von Mises' equivalent stress), $p=\frac{1}{3} \sigma: \boldsymbol{I}$ the hydrostatic stress, $E$ and $\nu$ the isotropic elastic constants. Parameters $\alpha, \sigma_{0}$ and $n$ govern the (fully incompressible) nonlinear part of the material response. All parameters describing the response of the aluminum alloy sheets are assumed to be known a priori with sufficient accuracy.

\subsection{Cohesive layer}

To describe the joint response under mixed-mode loading conditions, a modified version of the Xu-Needleman exponential law (Xu and Needleman, 1993), rooted in the atomistic description of binding energies, is chosen. This law, formulated for a zero-thickness interface, specifies a holonomic relationship between the tractions $\boldsymbol{T}$ on the interface, and the corresponding displacement discontinuity vector $\boldsymbol{\Delta}$. Normal and tangential components of traction and displacement vectors are denoted by $T_{n}$ and $T_{t}, \Delta_{n}$ and $\Delta_{t}$, respectively. Symbols $\phi_{n}$ and $\phi_{t}$ designate the works-of-separation (for a unit surface) under pure Mode I (peel) and Mode II (shear), respectively. The (non-dimensional) ratio between the tangential and normal works-ofseparation is denoted by $r=\phi_{t} / \phi_{n}$. Originally, the $\mathrm{Xu}-\mathrm{Needleman}$ interface response assumes that the works-of-separation $\phi_{n}$ and $\phi_{t}$ are equal $(r=1)$. 
However, in the case of structural adhesives the ratio $r$ generally lies in the range $3 \leq r \leq 6$ (Valoroso and Champaney, 2006). van den Bosch et al. (2006) proposed an extension of the $\mathrm{Xu}$-Needleman interface law that allows for different normal and tangential works-of-separation

$$
\begin{gathered}
T_{n}=\frac{\phi_{n}}{\delta_{n}}\left(\frac{\Delta_{n}}{\delta_{n}}\right) \exp \left(-\frac{\Delta_{n}}{\delta_{n}}\right) \exp \left(-\frac{\Delta_{t}^{2}}{\delta_{t}^{2}}\right) \\
T_{t}=\frac{2 \phi_{t}}{\delta_{t}}\left(\frac{\Delta_{t}}{\delta_{t}}\right)\left(1+\frac{\Delta_{n}}{\delta_{n}}\right) \exp \left(-\frac{\Delta_{n}}{\delta_{n}}\right) \exp \left(-\frac{\Delta_{t}^{2}}{\delta_{t}^{2}}\right)
\end{gathered}
$$

The above cohesive relationship is governed by four parameters, namely, $\phi_{n}$, $\phi_{t}, \delta_{n}$ and $\delta_{t}$ that describe the works-of-separation (per unit surface) and the characteristic lengths under mode I and mode II conditions, respectively. Although a sequence of images will be used for which the mode mixity is not constant, the experienced range of mixity is too narrow to lead to a robust determination of these four parameters as a whole. Complementary results from fracture tests under different loading conditions should be considered (e.g., pure mode I or mode II data). However, if the ratio $r$ is prescribed as an a priori known information, then the problem turns out to be sufficiently well posed for a robust determination of the three remaining free parameters (Section 3.5). Due to the significant scatter usually observed in the mechanical response of adhesive joints, this externally prescribed value is considered as a viable route to follow. In the following, the three unknown parameters governing the adhesive layer behavior are gathered in a vector form, $\boldsymbol{X}=\left\{\phi_{n}, \delta_{n}, \delta_{t}\right\}^{T}$.

\subsection{Extension to finite-thickness interfaces}

Zero-thickness representation of cohesive interfaces is widely adopted in computational mechanics to simulate quasi-brittle fracture processes at dif- 


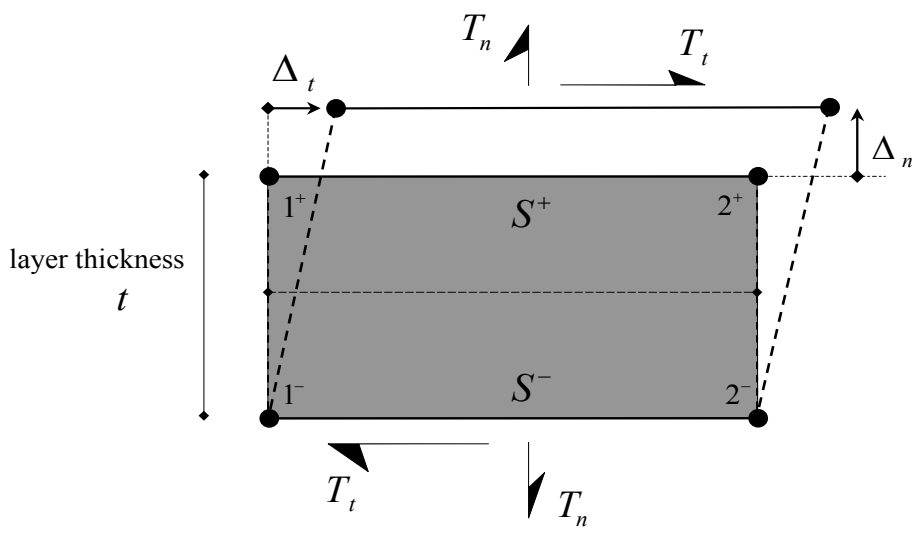

Figure 6: Interface element with a finite thickness.

ferent scales (Bolzon et al., 2002; Shet and Chandra, 2004; Andena et al., 2006). However, this simplification cannot be made in the present application. First, from a geometrical point of view, the thickness of the adhesive layer (approximately $60 \mu \mathrm{m}$ ) is not negligible with respect to the overall size of the modeled region at the microscale (about $600 \mu \mathrm{m}$ for the shortest size of the ROI). Second, elastic strains are expected to influence the overall response of the considered sub-domain. While the crack propagates, a part of the adhesive joint is compressed (a slightly reduced layer thickness can be noticed on the left side of the ROI in Figure 4).

In this study, the adhesive layer is modeled by means of finite-thickness interface elements. A simplified kinematics is assumed by describing two dominating deformation modes, namely, peel (normal) and shear (tangen- 
tial), both constant through the thickness (Figure 6). From a mechanical standpoint, corroborated by experimental evidences, an adhesive layer is expected to behave differently from the adhesive as a bulk material (Högberg, 2006). The constitutive law of the adhesive layer, even with finite thickness, is thus expressed to be a function of normal, $\Delta_{n}$, and tangential, $\Delta_{t}$, displacements. The cohesive element adopted herein can be regarded as an interface line element, with an initial gap between opposite nodes, otherwise coincident.

With such an interface element, let us consider a suitable partition for the nodal displacement vector $\boldsymbol{U}$, namely $\boldsymbol{U}=\left\{\boldsymbol{U}^{-}, \boldsymbol{U}^{+}\right\}^{T}$, where superscripts $(\cdot)^{ \pm}$denote corresponding nodes belonging to the upper and lower element side, respectively (Figure 6). The relative displacement vector is expressed as

$$
\boldsymbol{\Delta}=\left\{\begin{array}{c}
\Delta_{n} \\
\Delta_{t}
\end{array}\right\}=\left[-\boldsymbol{R}^{T} \boldsymbol{N} \boldsymbol{U}^{-}, \boldsymbol{R}^{T} \boldsymbol{N} \boldsymbol{U}^{+}\right]=\left[-\boldsymbol{B}_{s} \boldsymbol{U}^{-}, \boldsymbol{B}_{s} \boldsymbol{U}^{+}\right]=\boldsymbol{B} \boldsymbol{U}
$$

where $\boldsymbol{B}$ is the compatibility operator, and $\boldsymbol{R}$ the orthogonal rotation matrix, transforming the global coordinate system to the local one (in the present case $\boldsymbol{R}=\boldsymbol{I}$ ). Shape functions, gathered in matrix $\boldsymbol{N}$, vary linearly along the element length, and are constant through the layer thickness. The traction $\boldsymbol{T}$ at the joint interface is expressed as a function of the relative displacement vector $\boldsymbol{\Delta}$ [Equations (2a)-(2b)]. At each interface element $e$ in the adopted discretization, the vector of internal nodal forces, $\boldsymbol{F}_{i n t}^{(e)}$, and the tangent stiffness matrices, $\boldsymbol{K}_{\boldsymbol{u}}^{(e)}$, are defined as (Goyal et al., 2004)

$$
\boldsymbol{F}_{\text {int }}^{(e)}=\left\{\begin{array}{c}
-\boldsymbol{F}_{s} \\
\boldsymbol{F}_{s}
\end{array}\right\} \quad \boldsymbol{F}_{s}=\int_{-1}^{+1} \boldsymbol{B}_{s}^{T} \boldsymbol{T}|J| d \xi
$$




$$
\boldsymbol{K}_{\boldsymbol{u}}^{(e)}=\left[\begin{array}{cc}
\boldsymbol{K}_{s} & -\boldsymbol{K}_{s} \\
-\boldsymbol{K}_{s} & \boldsymbol{K}_{s}
\end{array}\right] \quad \boldsymbol{K}_{s}=\int_{-1}^{+1} \boldsymbol{B}_{s}^{T} \mathcal{K}_{\boldsymbol{u}} \boldsymbol{B}_{s}|J| d \xi
$$

where $\xi$ is the iso-parametric line coordinate, $|J|$ the jacobian (here constant) of the iso-parametric (line) mapping, $\mathcal{K}_{\boldsymbol{u}}=\partial \boldsymbol{T} / \partial \boldsymbol{\Delta}^{T}$ the material tangent stiffness matrix. One-dimensional integrals in Equations (4) and (5) are computed via a Newton-Cotes scheme (with collocation points at the two ends of the master element midline, for $\xi= \pm 1$ ). The formulation (3-5) allows one to obtain an interface response that is independent of the layer thickness, thus avoiding the scaling of work-of-separation, which is instead required when the dissipated energy is smeared over the element area (Balzani and Wagner, 2008).

The cohesive law (2a-2b) is further modified to take into account compressive normal tractions $\left(T_{n}<0\right)$ generated within the finite-thickness elements. The situation predicted by the cohesive relationship for $\Delta_{n} \rightarrow 0^{+}$, Equations (2a)-(2b), is prolonged when $\Delta_{n}<0$ as follows: (i) along the normal direction $n$, the adhesive response is governed by the tangent stiffness matrix at the origin, as if it were linearly elastic, in a decoupled way with respect to the tangential response; (ii) along the tangential direction $t$, a zero value of $\Delta_{n}$ is assumed in Equation (2b) and the tangential response $T_{t}$ is the one resulting from a pure mode II. Thus, in the present study, Equations (2a)-(2b) specify the adhesive response for $\Delta_{n} \geq 0$, whereas for $\Delta_{n}<0$ the following de-coupled equations hold

$$
\begin{gathered}
T_{n}=\frac{\phi_{n}}{\delta_{n}^{2}} \Delta_{n} \\
T_{t}=\frac{2 \phi_{t}}{\delta_{t}}\left(\frac{\Delta_{t}}{\delta_{t}}\right) \exp \left(-\frac{\Delta_{t}^{2}}{\delta_{t}^{2}}\right)
\end{gathered}
$$


This a priori simplification of the adopted model for $\Delta_{n}<0$ assumes no debonding in compression within the adhesive layer, friction or other kinds of coupling between tangential and normal tractions.

\subsection{Results}

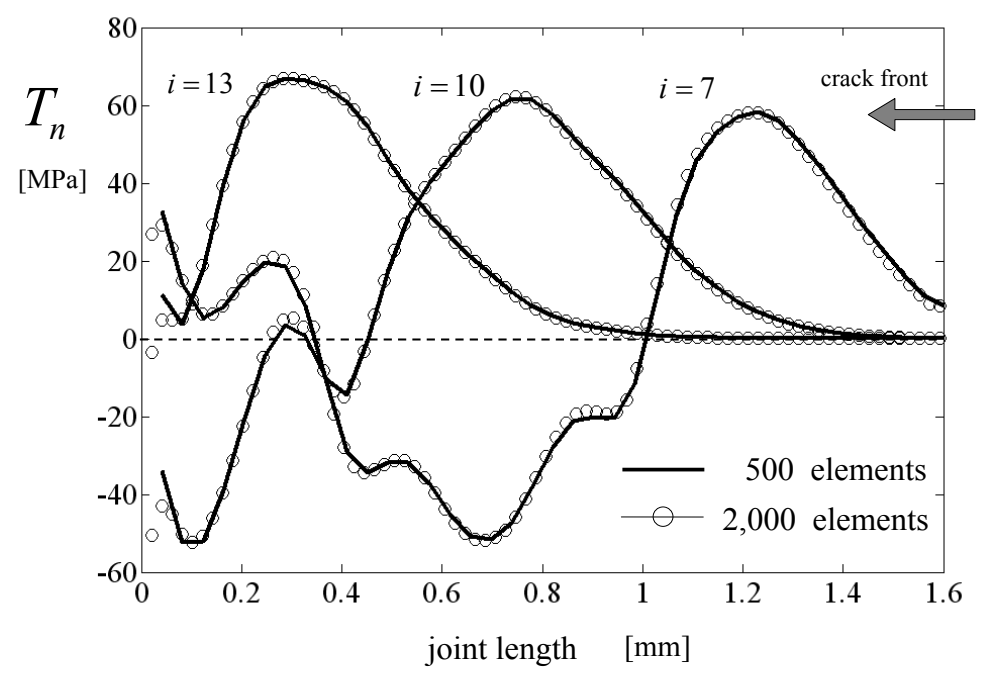

Figure 7: Profiles of normal tractions along the modeled interface, at different measurement instants $i$, computed with coarse (solid line) and fine (open circles) meshes.

The plane model adopted to simulate the mechanical response of the considered sub-domain is made of 570 finite elements, and the adhesive layer is discretized by means of 39 cohesive elements. Plane stress conditions are assumed since kinematic measurements provided by DIC concern the free surface of the specimen. The computer code for the nonlinear FE analyses and computation of parameter sensitivities was entirely developed in a 
Matlab ${ }^{\circledR}$ environment (Bonnet and Frangi, 2006), fully consistent with the DIC software in terms of mesh and shape functions.

It is worth noting that because of measurement uncertainties, the element size cannot be decreased at will since it will be accompanied by higher measurement uncertainties (Besnard et al., 2006). In the present case, an element size equal to 16 pixels (or $30.9 \mu \mathrm{m}$ ) is a compromise between uncertainty level and spatial resolution of the DIC procedure. To evaluate the mechanical effect of what may appear as a rather coarse mesh (i.e., 500 elements), a comparison is carried out with a finer mesh (using 2000 elements). Figure 7 shows the normal traction profiles along the modeled joint interface computed with the coarse and fine discretizations. The two profiles are very close. This result shows that the constraints imposed by DIC do not degrade significantly the results in terms of traction profiles since the measurements are performed close enough to the cohesive zone.

Twenty digital images are considered to analyze the debonding process within the ROI. Full field measurements are available at $m=19$ measurement instants, since the first image $(i=0)$ is the reference. Each forward analysis requires approximately 15 minutes on a standard PC (2.2 GHz Quad Core, and 3 Gigabyte RAM). The finite element simulation of the debonding process within the monitored sub-domain is shown in Figure 8. For those results, a fixed ratio of $r=6$ was chosen. Displacements provided by DIC along the boundary of the monitored zone, $\boldsymbol{u}_{\partial \Omega i}^{\exp }$, are prescribed at different instants $i(i=1, \ldots, 19)$ during the test. Figure 8 shows deformed meshes for four different steps of the debonding process.

The nonlinear relationship between the model parameters for the adhesive 

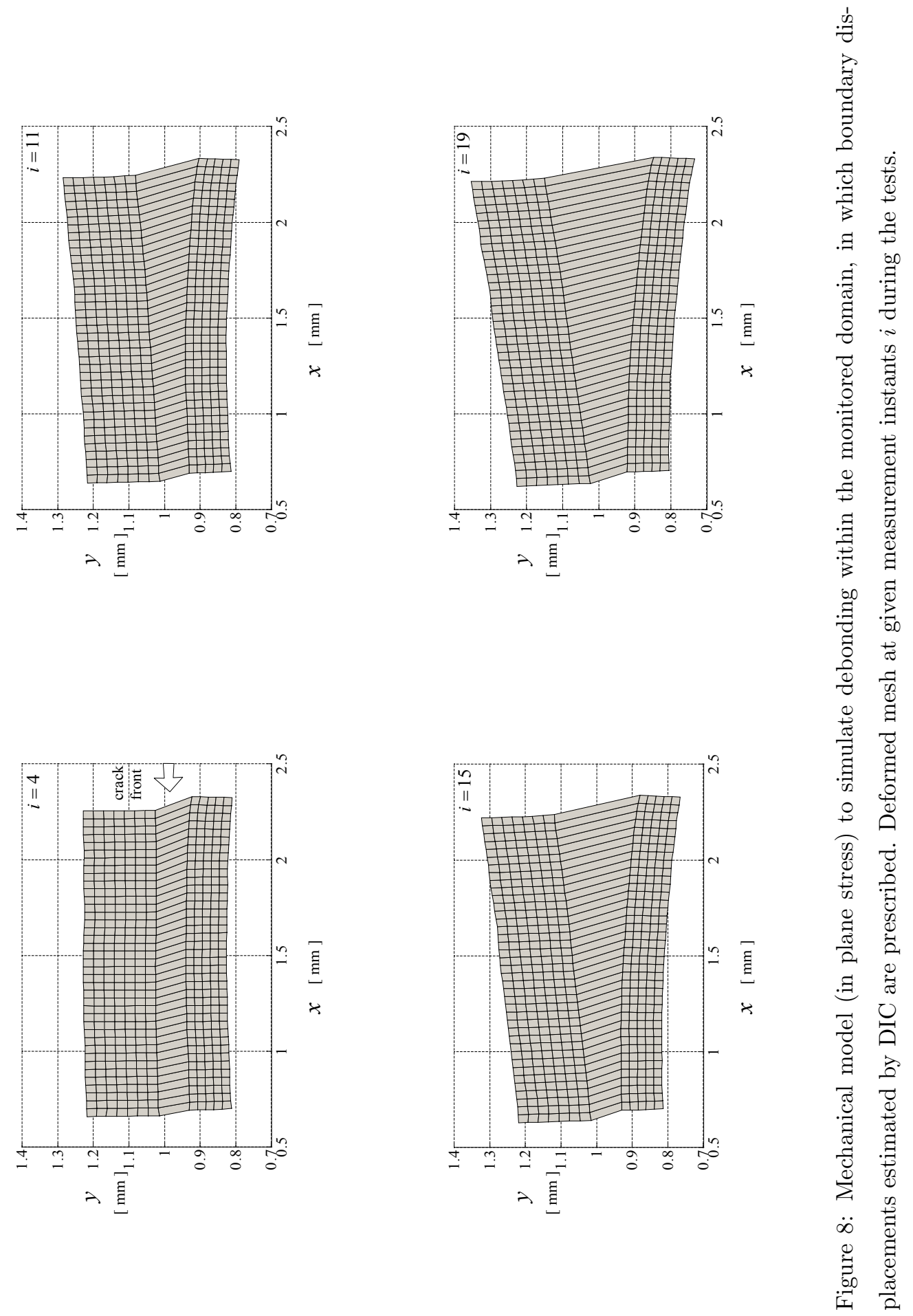
layer $\boldsymbol{X}$ and the displacements at measurement instant $i$ inside the ROI, adopted as measurable quantities, namely $\boldsymbol{u}_{\Omega i}^{\text {comp }}$, is usually referred to as forward or direct operator (as opposed to inverse), and denoted by

$$
\boldsymbol{u}_{\Omega i}^{\mathrm{comp}}=\boldsymbol{H}_{i}\left(\boldsymbol{X} ; \boldsymbol{u}_{\partial \Omega i}^{\mathrm{exp}}\right) \quad i=1, \ldots, m \equiv 19
$$

Even for a preliminary validation of the inverse procedure on the basis of pseudo-experimental (i.e., synthetic) data, the prescribed kinematic boundary conditions are exclusively those estimated by DIC, namely $\boldsymbol{u}_{\partial \Omega i}^{\exp }$.

\subsection{Sensitivity analysis}

The aim of the sensitivity analysis is to determine which parameters of the chosen model can be identified with the present experimental configuration. Derivatives of the displacement field $\boldsymbol{u} \equiv\left\{u_{x}, u_{y}\right\}^{T}$ with respect to the model parameters to identify $\boldsymbol{X}$ represent a key ingredient of many identification procedures. Strategies to compute such quantities are commonly referred to as "sensitivity analyses" (Kleiber et al., 1997; Micharelis et al., 2006). A simple and effective method is adopted based on the direct differentiation of the governing equations (i.e., DDM strategy). The application of DDM to the present problem takes advantage from the holonomy hypothesis assumed for the nonlinear constitutive equations (for the substrates and the adhesive layer), and it is implemented in the same FE code employed for forward simulations. Let us consider the discretized finite element residual equations at the $n$-th loading step

$$
\boldsymbol{R}^{n}=\boldsymbol{R}^{n}\left(\boldsymbol{U}^{n}(\boldsymbol{X}), \boldsymbol{X}\right)=\mathbf{0}
$$

Equation (8) holds at equilibrium states, and displacements are computed by Newton-Raphson iterations. Due to the holonomy assumption, residuals 

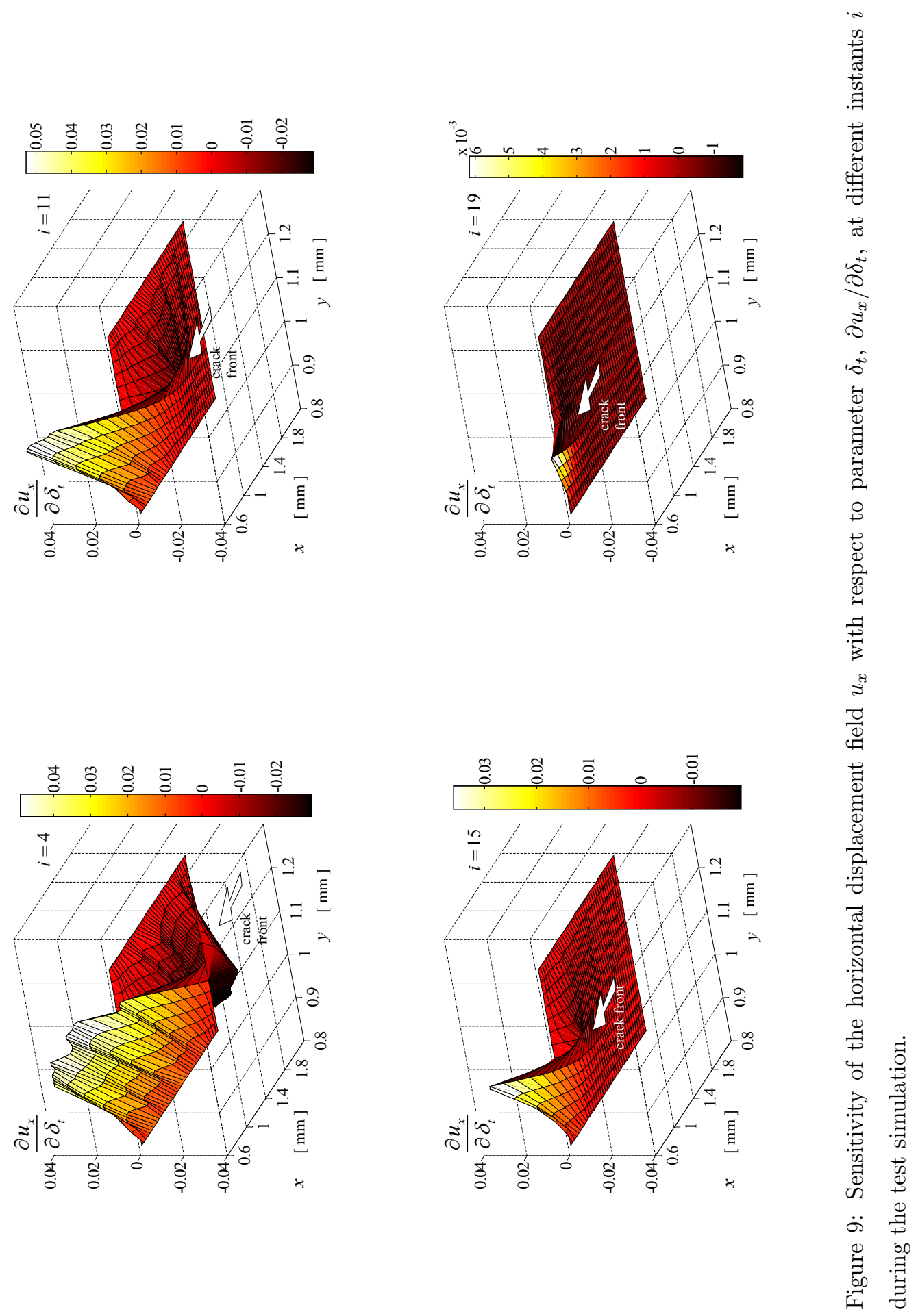
at the current $n$-th step $\boldsymbol{R}^{n}$ depend only on the current vector of nodal displacement $\boldsymbol{U}^{n}$, and on model parameters. The resulting displacement field inside the domain is affected by perturbations of the adhesive properties (sensitivities vanish where displacements are prescribed). By differentiating the above residual equation (8), the following relationship is satisfied

$$
\frac{\partial \boldsymbol{R}^{n}}{\partial \boldsymbol{U}^{n T}} \frac{\partial \boldsymbol{U}^{n}}{\partial \boldsymbol{X}^{T}}=-\frac{\partial \boldsymbol{R}^{n}}{\partial \boldsymbol{X}^{T}}
$$

Equation (9) provides parameter sensitivities for any steady-state mechanical problem (Micharelis et al., 2006). In the present context, residual equations over the ROI $\Omega$ particularize as

$$
\begin{gathered}
\boldsymbol{R}^{n}=\int_{\Omega} \boldsymbol{B}^{T} \boldsymbol{\sigma}^{n}\left(\boldsymbol{U}^{n}(\boldsymbol{X}), \boldsymbol{X}\right) d \Omega-\boldsymbol{F}_{\text {ext }}=\mathbf{0} \\
\frac{\partial \boldsymbol{R}^{n}}{\partial \boldsymbol{U}^{n T}}=\int_{\Omega} \boldsymbol{B}^{T} \frac{\partial \boldsymbol{\sigma}^{n}}{\partial \boldsymbol{U}^{n T}} d \Omega=\boldsymbol{K}_{\boldsymbol{u}} \quad \frac{\partial \boldsymbol{R}^{n}}{\partial \boldsymbol{X}^{T}}=\int_{\Omega} \boldsymbol{B}^{T} \frac{\partial \boldsymbol{\sigma}^{n}}{\partial \boldsymbol{X}^{T}} d \Omega
\end{gathered}
$$

where $\boldsymbol{F}_{\text {ext }}, \boldsymbol{B}$ and $\boldsymbol{\sigma}^{n}$ denote the vector of external forces, the compatibility matrix and the current value of local stress tensor, respectively. An analogy can be proposed between the linearized form of finite element equations, solved iteratively to compute Newton corrections, and the sensitivity analysis problem (9). Both problems are linear with respect to the unknowns and are governed by the assembled tangent matrix $\boldsymbol{K}_{\boldsymbol{u}}$. For this reason, the RHS of Equations (9) and (10) is referred to as "pseudo-load" vector. From a numerical standpoint, parameter sensitivities are easily computed at the end of each loading step of the finite element analysis, when convergence by Newton iterations is achieved, by solving only the linear system (9), irrespectively of the parameter vector dimension $n_{X}$. Since the tangent stiffness matrix is already available at each Newton iteration, the sensitivity analysis requires only the pseudo-load vector as additional datum. Such a vector 
is computed by assembling contributions from each element, analogously to what is performed for vector $\boldsymbol{F}_{\text {int }}$ gathering the internal forces. The above strategy leads to faster identification procedures, thereby avoiding numerous forward analyses required by finite differences schemes.

Figure 9 shows the sensitivity of horizontal displacement fields $u_{x}$ with respect to the parameter $\delta_{t}$, computed by the DDM at different measurement instants $i$ during the test. In spite of the noisy environment, at the beginning of the test the above parameter sensitivity is significant over almost the whole sub-domain, even if it peaks close to the adhesive layer $(i=4)$. As the crack propagates, horizontal measurements are less sensitive to such parameter, and non-vanishing sensitivities tend to concentrate around the adhesive joint. The peak values move toward the sound part of the adhesive layer, and indicate approximately the current location of the process zone within the adhesive joint $(i=11,15,19)$. Last, it is worth noting that displacement sensitivities with respect to parameter variations $\delta_{n} / \overline{\delta_{n}}$ and $\delta_{t} / \overline{\delta_{t}}$, where overlined quantities denote reference values, are significantly higher (especially the former) than those concerning the normal work-of-separation $\phi_{n}$. This result suggests not to add a further unknown by allowing ratio $r$ to vary.

\section{Reconstruction of interface tractions via parameter identifica- tion}

Since measured boundary conditions are used, and the pictures at hand (i.e., with a poor texture) will induce high measurement uncertainties, the identification technique needs to be robust enough to allow for low signal / 
noise ratios. The optimal parameters $\widehat{\boldsymbol{X}}$ are obtained as a minimization of a suitable discrepancy norm (Tarantola, 1987; Maier et al., 2005). Such a function quantifies the discrepancy between measured quantities (i.e., displacement of inner nodes of the ROI provided by DIC), and those computed as functions of the unknown model parameters. Let $m$ be the number of available measurement instants, $n_{n}$ the number of nodal displacements selected. The adopted objective function is the least-squares norm

$$
\begin{aligned}
\widehat{\boldsymbol{X}} & =\arg \min _{\boldsymbol{X}}\left\{\omega(\boldsymbol{X})=\sum_{i=1}^{m} \omega_{i}(\boldsymbol{X})\right\} \\
\omega_{i}(\boldsymbol{X}) & =\frac{1}{\left(W_{x}^{i}\right)^{2}} \sum_{j=1}^{n_{n}}\left[u_{x}^{\exp }-u_{x}^{\mathrm{comp}}(\boldsymbol{X})\right]_{j}^{2}+\frac{1}{\left(W_{y}^{i}\right)^{2}} \sum_{j=1}^{n_{n}}\left[u_{y}^{\exp }-u_{y}^{\text {comp }}(\boldsymbol{X})\right]_{j}^{2}
\end{aligned}
$$

where weights $W_{x}^{i}$ and $W_{y}^{i}$ at each measurement instant $i$ are defined as the maximum displacements (in absolute value, and after the subtraction of the average rigid body motions) measured at that instant, along the horizontal and vertical direction, respectively. The contributions of all $m$ discrepancies $\omega_{i}$ are additively included in a unique scalar objective function to minimize. The discrepancy between the experimental and computed displacements is based of $n_{n}=80$ nodal values and $m=19$ measurement instants, concerning both aluminum alloy strips. The number of used experimental data points, $m \times n_{r}=19 \times 80=1520$, is to be compared to the number of unknowns, 3 . This large ratio is responsible for the robustness of the procedure. 


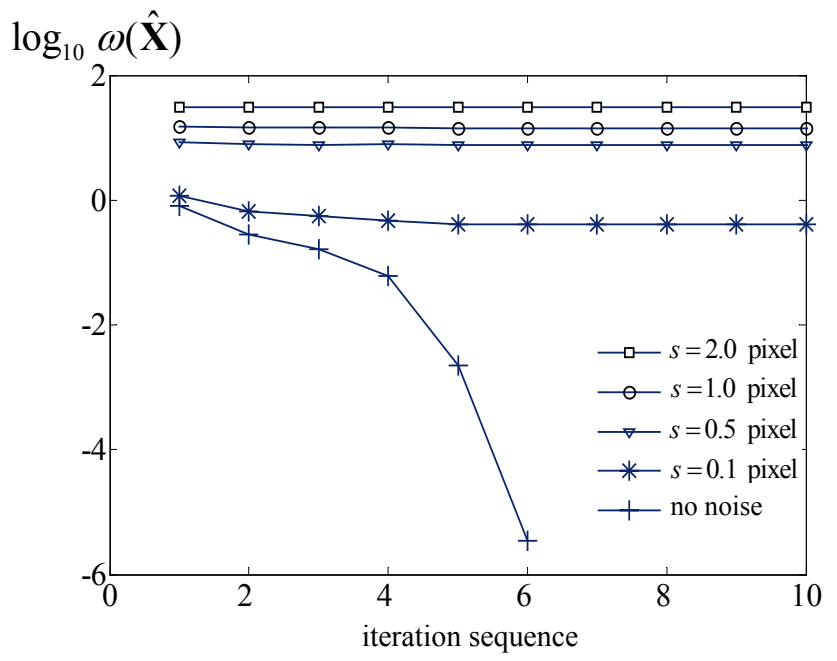

Figure 10: Validation of the identification procedure on the basis of artificial data. Minimization process of the objective function by Trust Region algorithm, in the presence of a Gaussian (non-correlated) noise on measured displacements, with increasing standard deviation $s$, namely $s=0,0.1,0.6,1$ and 2 pixels.

\subsection{Artificial data}

The displacements inside the discretized domain $\Omega$ are generated numerically by using an a priori assumed parameter set $\overline{\boldsymbol{X}}$. The parameter estimates $\widehat{\boldsymbol{X}}$ resulting from the identification procedure are thus compared with the original set, and the error is assessed. The reference parameters are $\overline{\phi_{n}}=1.0 \mathrm{~N} \mathrm{~mm}^{-1}, \overline{\delta_{n}}=5 \mu \mathrm{m}$ and $\overline{\delta_{t}}=50 \mu \mathrm{m}$. This parameter set specifies an adhesive layer capable of developing maximum normal and tangential tractions equal to $T_{n \text { max }}=74 \mathrm{MPa}$ and $T_{t \text { max }}=103 \mathrm{MPa}$, under pure mode I and II, respectively. The mechanical parameters for the aluminum alloy sheet are as follows: Young's modulus $E=72 \mathrm{GPa}$, Poisson's ratio $\nu=0.33$, 
yield stress $\sigma_{0}=180 \mathrm{MPa}$, yield offset $\alpha=0.06$ and hardening exponent $n=7$.

The minimization in the parameter space of the objective function $\omega(\boldsymbol{X})$ is performed through gradient-based (first-order) algorithms. The most recent Trust Region Method (Coleman and Li, 1996; Matlab, 2003) allows for box-constraints by an interior-point strategy. This provision is particularly important when an unknown model parameter entering constitutive models and input of finite element codes belong to a feasible interval, and the initialization set is not necessarily close to the minimum point.

To validate the above procedure, first artificial data are considered with no noise. In this situation, the three parameters are estimated correctly in a few iterations, up to machine precision. No dependence of the parameter estimates $\widehat{\boldsymbol{X}}$ on the algorithm initialization, which might have suggested the presence of secondary minima, was observed. Thereafter, to investigate the robustness of the inverse analysis procedure, displacements inside the subdomain $\Omega$ are corrupted by means of an additive, uncorrelated (over time and space) Gaussian noise $\boldsymbol{e}$

$$
\boldsymbol{y}_{i}^{\exp }=\boldsymbol{H}_{i}\left(\boldsymbol{X} ; \boldsymbol{u}_{\partial \Omega i}^{\exp }\right)+\boldsymbol{e}_{i}
$$

with zero average and standard deviation $s$. For the sake of simplicity, prescribed boundary displacements provided by DIC, namely $\boldsymbol{u}_{\partial \Omega i}^{\exp }$, which are already affected by measurement uncertainties, are left unchanged. If the parameter estimates $\widehat{\boldsymbol{X}}$ resulting from the inverse procedure are far from the correct values (a conventional error threshold of $15 \%$ is given), the inverse problem is expected to be ill-posed, and non-identifiability should be invoked with regard to one or more parameters. Values of standard devia- 
Table 1: Identified parameters as functions of the standard uncertainty level $s$ of the displacement data.

\begin{tabular}{|c|c|c|c|c|}
\hline$s$ (pixels) & 0.1 & 0.6 & 1.0 & 2.0 \\
\hline$\phi_{n}\left(\mathrm{~N} \mathrm{~mm}^{-1}\right)$ & $1.0(2 \%)$ & $0.9(10 \%)$ & $1.0(3 \%)$ & $0.9(6 \%)$ \\
$\delta_{n}(\mu \mathrm{m})$ & $5.0(1 \%)$ & $5.1(1 \%)$ & $5.6(12 \%)$ & $7.4(48 \%)$ \\
$\delta_{t}(\mu \mathrm{m})$ & $50(1 \%)$ & $48(5 \%)$ & $52(3 \%)$ & $49(2 \%)$ \\
$\omega(\widehat{\boldsymbol{X}})$ & 0.4 & 7.5 & 14.3 & 30.6 \\
\hline
\end{tabular}

tions considered for white noise are as follows: $s=0.1,0.6,1.0,2.0$ pixels. Table 1 shows the parameter estimates resulting from data with increasing level of noise, together with their percentage error (in parentheses) and values of objective function at the minimum point $\widehat{\boldsymbol{X}}$. Values of the objective function at the minimum point, $\omega(\widehat{\boldsymbol{X}})$, increase significantly with the noise level (Figure 10). In the presence of noise levels with a standard deviation $s \leq 1.0$ pixel, the resulting parameter estimates $\widehat{\boldsymbol{X}}$ provided by the above inverse procedure are rather acceptable (i.e., the relevant percentage error does not exceed the chosen threshold of $15 \%$ ). For $s \geq 2$ pixels instead, the parameter $\delta_{n}$ is no longer identifiable by the above numerical procedure. It is worth emphasizing that a standard deviation $s=2$ pixels implies that the single Gaussian perturbation term belongs to the interval $e \in[-6,+6]$ pixels, with a probability of $99 \%$. With reference to the DIC procedure, such a noise intensity on kinematic data is very high (Besnard et al., 2006), and therefore the inverse procedure can be regarded as sufficiently robust in the present case. 


\subsection{Experimental data}

Experimental data of Section 2 are considered. Through the bilinear shape functions defined over each finite element, kinematic data provided by the DIC algorithm are interpolated at the discretization nodes belonging to the mechanical model. Parameter estimates obtained by the inverse procedure are $\phi_{n}=2.5 \mathrm{~N} \mathrm{~mm}^{-1}, \delta_{n}=8.5 \mu \mathrm{m}, \delta_{t}=85 \mu \mathrm{m}$, and hence $\phi_{t}=r \phi_{n}=9.8 \mathrm{~N} \mathrm{~mm}^{-1}$. The value of the objective function at the mini-

mum point is equal to $\omega(\widehat{\boldsymbol{X}})=7.5$. It is worth noting that the latter has a level close to the noisy case resting on independent Gaussian perturbations, with standard deviation $s=0.6$ pixel (Table 1) for which the identification was deemed acceptable.

Although the cohesive model adopted herein describes a nonlinear behavior of the adhesive layer, useful indications are obtained concerning the elastic properties of the adhesive layer. From the estimated parameters, the (de-coupled) tangent stiffness at the origin $\left(\Delta_{n}, \Delta_{t} \rightarrow 0\right)$ reads

- along the normal direction, $\partial T_{n} / \partial \Delta_{n}=\phi_{n} / \delta_{n}^{2}=34.1 \mathrm{~N} \mathrm{~mm}^{-3}$,

- and along the tangential direction, $\partial T_{t} / \partial \Delta_{t}=2 r \phi_{n} / \delta_{t}^{2}=2.7 \mathrm{~N} \mathrm{~mm}^{-3}$.

Such values have to be regarded as effective properties of the adhesive layer at the microscale, and are expected to be different from those of the bulk material (since the layer thickness is $t \simeq 60 \mu \mathrm{m}$ ). If for the bulk epoxy adhesive Young's modulus $E_{a d h} \simeq 1 \mathrm{GPa}$ and Poisson's ratio $\nu_{a d h} \simeq 0.4$ are assumed, the stiffness values estimated above are comparable to $E_{a d h}(1-$ $\left.\nu_{a d h}\right) /\left[\left(1+\nu_{a d h}\right)\left(1-2 \nu_{a d h}\right) t\right] \simeq 36 \mathrm{~N} \mathrm{~mm}^{-3}$ and $E_{a d h} /\left[2\left(1+\nu_{a d h}\right) t\right] \simeq 5.9 \mathrm{~N}$ 

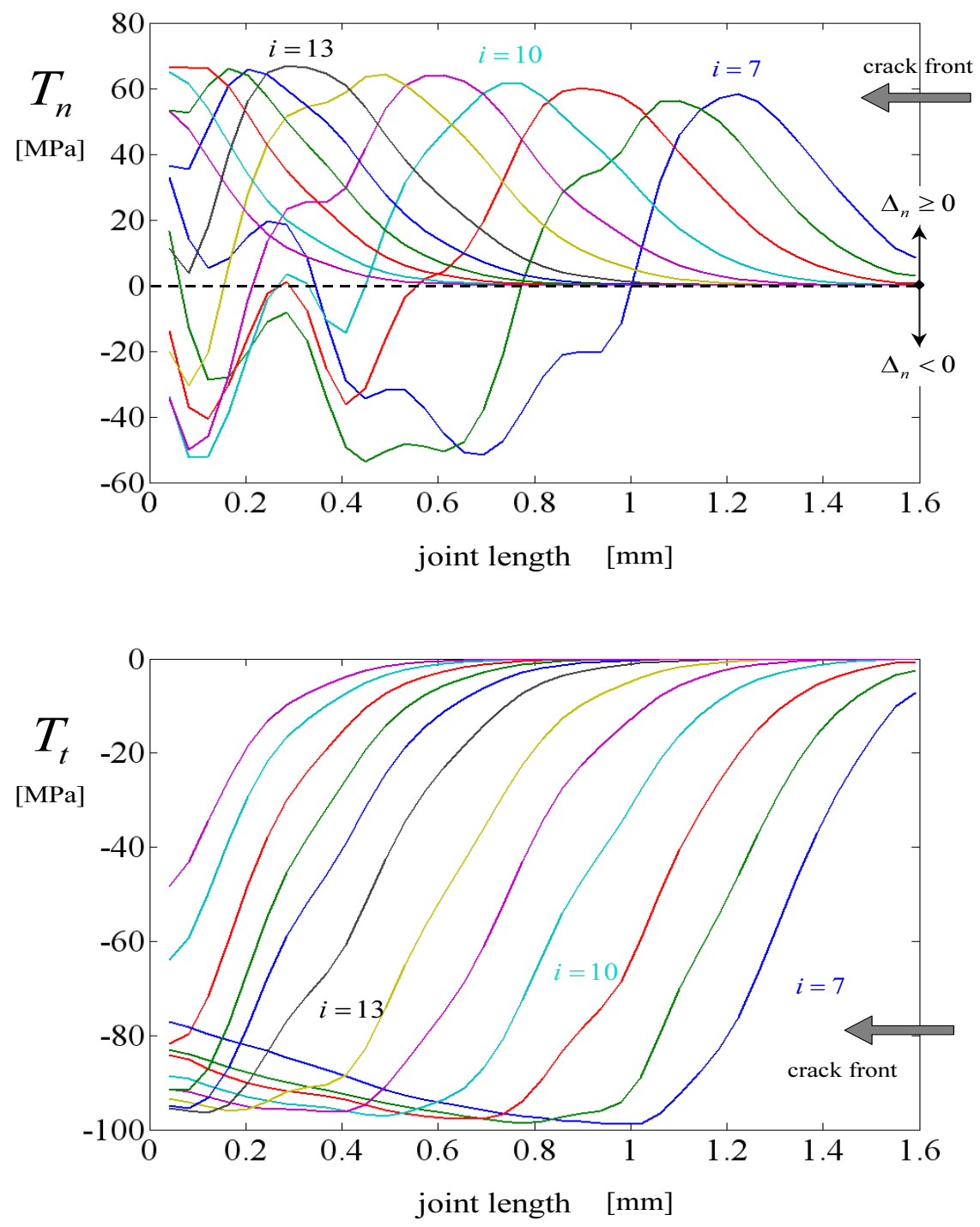

Figure 11: Change of normal and tangential traction profiles at the adhesive interface, $T_{n}$ and $T_{t}$, resp., re-constructed by the proposed identification procedure at different measurement instants $i$, with the a priori assumed ratio $r=4$. 
$\mathrm{mm}^{-3}$ (Högberg, 2006), along the normal and tangential direction, respectively.

Figure 11 shows the profiles of tangential and normal tractions along the adhesive layer computed by the identified model at different measurement instants $i(8 \leq i \leq 19)$ during the test. The profiles of normal tractions $T_{n}$ exhibit a classical bell shape with a softening branch for tensile values when $\Delta_{n} \geq 0$, while compressive values are observed in the remaining part of the adhesive layer $\left(T_{n}<0\right.$ when $\left.\Delta_{n}<0\right)$. During the debonding process, profiles of normal tractions $T_{n}$ translate progressively towards the left part of the monitored sub-domain as the crack propagates. Simultaneously, their peak values change, since the mode mixity varies continuously during the experiment along the adhesive layer. The traction-free part of the interface, corresponding to a macroscopic crack observable in digital images (see Figure 4), becomes larger, starting from the right end of the ROI, and the compressed part $\left(T_{n}<0\right)$ at the left side progressively reduces until it completely vanishes. As a consequence of measurement uncertainties, small values of tensile tractions $(\leq 10 \mathrm{MPa})$ are observed on the left end of the ROI. The profiles of tangential tractions $T_{t}$ appear to be smoother with respect to the normal ones, since the horizontal displacements detected by DIC and prescribed along the model boundaries are larger and yield better signal to noise ratios. Moreover, horizontal boundary displacements prescribed at each aluminum alloy strip exhibit a rather limited variation along the layer. Therefore, bell-shape profiles for tangential tractions are less obvious. The pre-peak response is clearly distinguished only at the first measurement instants. 

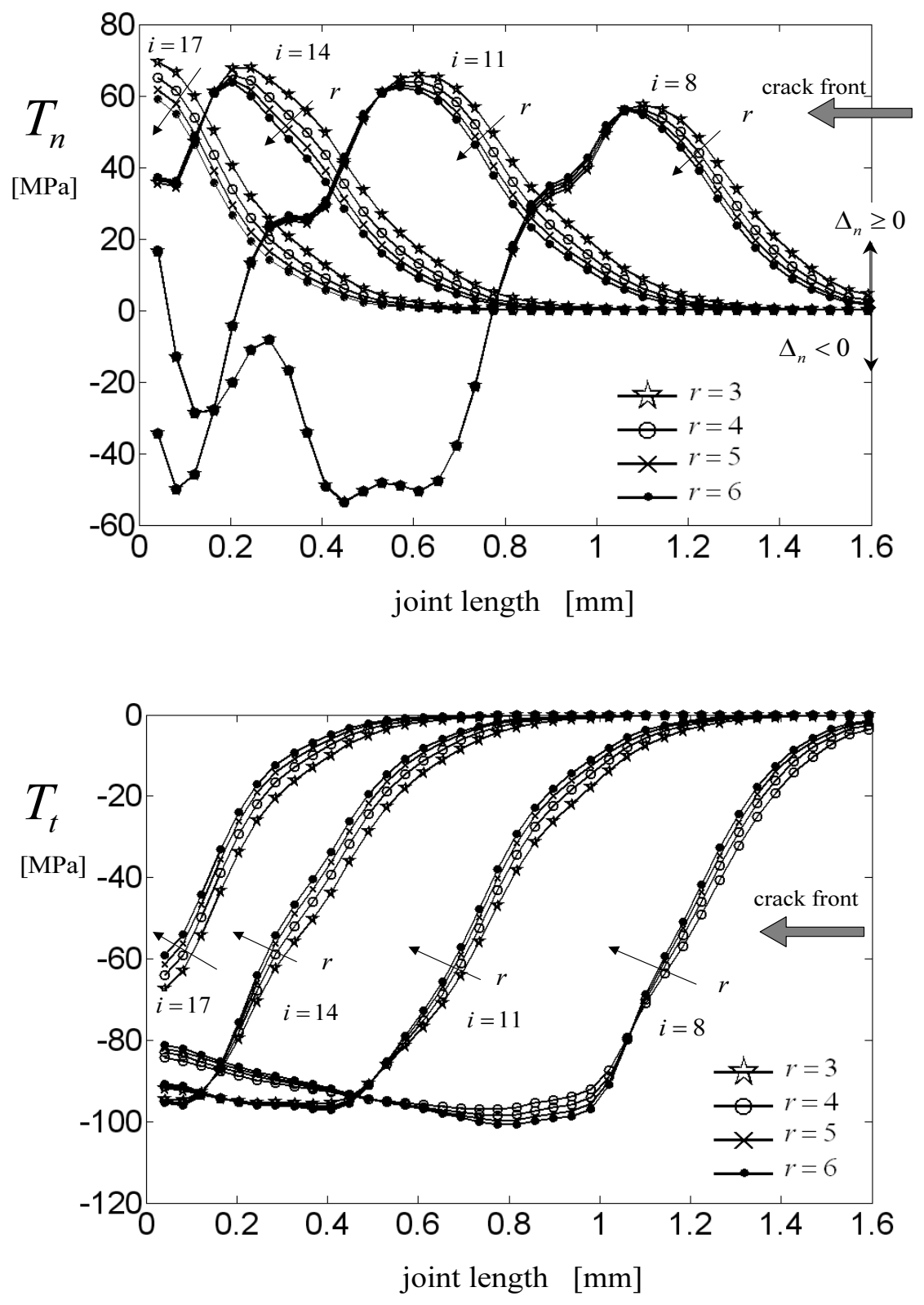

Figure 12: Interface tractions $T_{n}$ at measurement instants $i=8,11,14,17$, identified by the proposed inverse procedure for different values of the parameter $3 \leq r \leq 6$. 
These results show that the loading conditions generated at the microscale during the test proposed herein are rather severe in order to calibrate a mixedmode cohesive model. Mode mixity varies continuously during the debonding experiment along the adhesive layer. Interface tractions computed by the identified model (Figure 11) are reasonable from an engineering standpoint, when compared to experimental data on structural adhesives available in the literature (Högberg et al., 2006; Leffler et al., 2007; Hua et al., 2008) when taking into account the relevant layer thickness. Under pure mode I and mode II, the calibrated model predicts maximum tractions $T_{n \max }=$ $107 \mathrm{MPa}$ and $T_{t \max }=99 \mathrm{MPa}$. To assess the role played by the dimensionless

Table 2: Values of the different identified parameters as a function of the $r$ ratio.

\begin{tabular}{|c|c|c|c|c|}
\hline$r$ & 3.0 & 4.0 & 5.0 & 6.0 \\
\hline$\phi_{n}\left(\mathrm{~N} \mathrm{~mm}^{-1}\right)$ & 3.0 & 2.5 & 2.2 & 1.9 \\
$\delta_{n}(\mu \mathrm{m})$ & 9.4 & 8.5 & 7.9 & 7.5 \\
$\delta_{t}(\mu \mathrm{m})$ & 80 & 85 & 90 & 94 \\
\hline$\phi_{t}=r \phi_{n}\left(\mathrm{Nmm}^{-1}\right)$ & 9.1 & 9.8 & 10.6 & 11.4 \\
$T_{n \max }(\mathrm{MPa})$ & 118 & 107 & 99 & 94 \\
$T_{t \max }(\mathrm{MPa})$ & 97 & 99 & 101 & 103 \\
$\left.\left.\frac{\partial T_{n}}{\partial \Delta_{n}}\right|_{0}(\mathrm{GPa} \mathrm{mm})^{-1}\right)$ & 34 & 34 & 34 & 34 \\
$\left.\left.\frac{\partial T_{t}}{\partial \Delta_{t}}\right|_{0}(\mathrm{GPa} \mathrm{mm})^{-1}\right)$ & 2.8 & 2.7 & 2.6 & 2.6 \\
$\omega(\widehat{\boldsymbol{X}})$ & 7.5 & 7.5 & 7.5 & 7.5 \\
\hline
\end{tabular}

ratio $r=\phi_{t} / \phi_{n}$, the same identification process was repeated by assuming different values for $r$. Table 2 shows for each value of $r$ various parameter 
estimates. As expected from the sensitivity analysis, most values are virtually independent of $r$. Traction profiles $\left(T_{n}\right.$ and $\left.T_{t}\right)$ at meaningful measurement instants $(i=8,11,14,17)$ generated with the above parameter estimates are shown in Figure 12. It is worth emphasizing that such traction profiles are quite close to each other, even if values of the corresponding parameter sets $\widehat{\boldsymbol{X}}$ exhibit significant differences. Other quantities of mechanical interest, such as the maximum tensile tractions (not measured) under pure mode I, $T_{n \max }$, predicted by the model with the above estimates (Table 2), are also varying with $r$. Further experimental tests under different loading conditions are needed to tune properly the parameter $r$.

\section{Closing remarks and outlook}

In this study the mechanical properties of an adhesive layer in a GLARE assembly, manufactured with an industrial process, have been estimated through an inverse method based exclusively on full field displacements provided by DIC. Non-conventional tests were designed to generate mixed-mode fracture conditions within the adhesive layer. Displacements at the microscale estimated by DIC are adopted to specify Dirichlet boundary conditions for a small sub-domain, including part of the adhesive layer subjected to debonding and part of the aluminum alloy substrates.

Local kinematic measurements, provided by DIC in a format that is fully consistent with conventional finite element discretizations, enabled us to focus the inverse analysis on the sole ROI of the DIC analysis. This procedure allows one to model only a small part of the experiment since the effect of the remote boundary conditions is accounted for by the measured displacements 
on the boundary of the ROI. The inner displacements are then used to tune the parameters of the cohesive law. Kinematic data are thus adopted simultaneously to drive the mechanical simulation of the test (Dirichlet conditions prescribed along the external boundary), and as comparison term (inside the domain) included in the objective function. The parameters were identified and a good match was found between measured and computed (inner) displacements at the optimum point.

The analysis performed herein, and resting only on measured displacements, is quite general in terms of applicability. For instance, delamination in composite materials, friction and sliding in metal/metal or hybrid assemblies may be analyzed with the same type of procedure. Last, the measured displacement field may be enriched as in extended finite elements (Black and Belytschko, 1999; Moës et al., 1999) to better capture displacement discontinuities (Réthoré et al., 2007, 2008).

\section{Acknowledgements}

The Authors thank ALENIA for providing composite laminates investigated in a research project between LMT-Cachan and Politecnico di Milano, supported by the EU project "Knowledge-based Multicomponent Materials for Durable and Safe Performance" (KMM-NoE, NMP3-CT-2004-502243), and by the "young researcher fund 2006" of Politecnico di Milano. 


\section{References}

J. Abanto-Bueno and J. Lambros, Investigation of crack growth in functionally graded materials using digital image correlation, Eng. Fract. Mech. 69 (2002) 1695-1711.

J. Abanto-Bueno and J. Lambros, Experimental Determination of Cohesive Failure Properties of a Photodegradable Copolymer, Exp. Mech. 45 [2] (2005) 144-152.

L. Andena, M. Rink and J. G. Williams, Cohesive zone modelling of fracture in polybutene, Eng. Fract. Mech. 73 (2006) 476-485.

C. Balzani and W. Wagner, An interface element for the simulation of delamination in unidirectional fiber-reinforced composite laminates, Eng. Fract. Mech.[75(9)] (2008) 2597-2615.

G. Besnard, F. Hild and S. Roux, "Finite-element" displacement fields analysis from digital images: Application to Portevin-Le Châtelier bands, Exp. Mech. 46 (2006) 789-803.

T. Black and T. Belytschko, Elastic crack growth in finite elements with minimal remeshing, Int. J. Num. Meth. Eng. 45 (1999) 601-620.

G. Bolzon, R. Fedele and G. Maier, Parameter identification of a cohesive crack model by Kalman Filter, Comp. Meth. Appl. Mech. Eng. 191 (2002) 2947-2871.

M. Bonnet and A. Frangi, Analyse des solides déformables par la méthode des 
éléments finis, (Les Editions de l'Ecole Polytechnique, Palaiseau (France), 2006).

M. J. van den Bosch, P. J. G. Schreurs and M. G. D. Geers, An improved description of the exponential $\mathrm{Xu}$ and Needleman cohesive zone law for mixed-mode decohesion, Eng. Fract. Mech. 73 (2006) 1220-1234.

I. Chasiotis and W. G. Knauss, A New Microtensile Tester for the Study of MEMS Materials with the Aid of Atomic Force Microscopy, Exp. Mech. $42[1](2002)$ 51-57.

I. Chasiotis (edt.), Special issue on nanoscale measurements in Mechanics, Exp. Mech. 47 [1] (2007).

S. Cho, J. F. Cárdenas-García and I. Chasiotis, Measurement of Nanodisplacements and Elastic Properties of MEMS via the Microscopic Hole Method, Sensors and Actuators A 120 (2005) 163-171.

T. F. Coleman and Y. Li, An interior trust region approach for nonlinear minimization subject to bounds, SIAM J. Opt. 6 [2] (1996) 418-445.

A. Derewonko, J. Godzimirski, K. Kosiuczenko, T. Niezgoda and A. Kiczko, Strength assessment of adhesive-bonded joints, Comput. Mat. Sci. 43 [1] (2008) 157-164.

P. Forquin, L. Rota, Y. Charles and F. Hild, A Method to Determine the Toughness Scatter of Brittle Materials, Int. J. Fract. 125 [1] (2004) 171187. 
M. G. D. Geers, R. de Borst and T. Peijs, Mixed numerical-experimental identification of non-local characteristics of random-fibre-reinforced composites, Comp. Sci. Tech. 59 (1999) 1569-1578.

V. K. Goyal, E. R. Johnson and C. G. Davila, Irreversible constitutive law for modeling the delamination process using interfacial surface discontinuities, Comp. Struct. 65 (2004) 289-305.

F. Hild and S. Roux, Digital image correlation: From measurement to identification of elastic properties - A review, Strain 42 (2006) 69-80.

J. L. Högberg, Mixed mode cohesive law, Int. J. Fract. 141 (2006) 549-559.

J.L. Högberg and B.F. Sørensen and U. Stigh, Constitutive behaviour of mixed mode loaded adhesive layer, Int. J. Solids Struct.[44] (2006) 83358354.

Y. Hua, A. D. Crocombea, M. A. Wahaba and I. A. Ashcroft, Continuum damage modelling of environmental degradation in joints bonded with EA9321 epoxy adhesive, Int. J. Adhesion Adhesives 28 (2008) 302-313.

M. Kleiber, H. Antunez, T. D. Hien and P. Kowalczyk, Parameter Sensitivity in Nonlinear Mechanics. Theory and Finite Element Computations, (John Wiley \& Sons, New York (USA), 1997).

K. Leffler, K. S. Alfredsson and U. Stigh, Shear behaviour of adhesive layers, Int. J. Solids Struct. 44 (2007) 530-545.

J. Lemaitre and J.-L. Chaboche, Mechanics of Solid Materials, (Cambridge University Press, Cambridge (UK), 1990). 
G. Maier, M. Bocciarelli and R. Fedele, Some innovative industrial prospects centered on inverse analyses, in: Parameter Identification of Materials and Structures. CISM Lecture Notes., Z. Mróz and G. Stavroulakis, eds., (Springer Verlag, Wien, 2005), 47-73.

Matlab 6.5, Optimization Toolbox Manual, (The MathWorks Inc., 2003).

S. R. McNeill, W. H. Peters and M. A. Sutton, Estimation of stress intensity factor by digital image correlation, Eng. Fract. Mech. 28 [1] (1987) 101112 .

P. Michaleris, D. A. Tortorelli and C. A. Vidal, Tangent Operators and Design Sensitivity. Formulations for Transient Nonlinear Coupled Problems with Applications to Elasto-Plasticity, Int. J. Num. Meth. Eng. 37 (1994) 2471-2499.

N. Moës, J. Dolbow and T. Belytschko, A finite element method for crack growth without remeshing, Int. J. Num. Meth. Eng. 46 [1] (1999) 133150.

T. Pardoen, T. Ferracin, C. M. Landis and F. Delannay, Constraint effects in adhesive joint fracture, J. Mech. Phys. Solids 53 (2005) 1951-1983.

J. Réthoré, F. Hild and S. Roux, Shear-band capturing using a multiscale extended digital image correlation technique, Comp. Meth. Appl. Mech. Eng. 196 [49-52] (2007) 5016-5030.

J. Réthoré, F. Hild and S. Roux, Extended digital image correlation with crack shape optimization, Int. J. Num. Meth. Eng. 73 [2] (2008) 248-272. 
K. Salomonsson and T. Andersson, Modeling and parameter calibration of an adhesive layer at the meso level, Mech. Mat. 40 [1-2] (2008) 48-65.

J. P. Sargent, Durability studies for aerospace applications using peel and wedge tests, Int. J. Adhesion Adhesives 25 (2005) 247-256.

C. Shet and N. Chandra, Effect of the Shape of $T \delta$ Cohesive Zone Curves on the Fracture Response, Mech. Adv. Mat. Struct. 11 (2004) 249-275.

J. Sinke, Manufacturing of GLARE Parts and Structures, Appl. Compos. Mat. 10 (2003) 293305.

E. Soppa, P. Doumalin, P. Binkele, T. Wiesendanger, M. Bornert and S. Schmauder, Experimental and numerical characterisation of in-plane deformation in two-phase materials, Comput. Mat. Sci. 21 [3] (2001) 261-275.

B. F. Sørensen, E. K. Gamstedt, R. C. Østergaard and S. Goutianos, Micromechanical model of cross-over fibre bridging Prediction of mixed mode bridging laws, Mech. Mat. 40 (2008) 220-234.

C. Su, Y. J. Wei and L. Anand, An elasticplastic interface constitutive model: application to adhesive joints, Int. J. Plasticity 20 (2004) 2063-2081.

M. A. Sutton, W. Zhao, S. R. McNeill, J. D. Helm, R. S. Piascik and W. T. Riddel, Local crack closure measurements: Development of a measurement system using computer vision and a far-field microscope, in: Advances in fatigue crack closure measurement and analysis: second volume, STP 1343, R. C. McClung and J. C. Newman Jr., eds., (ASTM, 1999), 145-156. 
M. A. Sutton, S. R. McNeill, J. D. Helm and Y. J. Chao, Advances in TwoDimensional and Three-Dimensional Computer Vision, in: Photomechanics, P. K. Rastogi, eds., (Springer, Berlin (Germany), 2000), 323-372.

A. Tarantola, Inverse Problems Theory. Methods for Data Fitting and Model Parameter Estimation, (Elsevier Applied Science, Southampton (UK), 1987).

N. Valoroso and L. Champaney, A damage-mechanics-based approach for modelling decohesion in adhesively bonded assemblies, Eng. Fract. Mech. 73 (2006) 2774-2801.

A. Vlot, Glare, History of the Development of a New Aircraft Material, (Kluwer Academic Publishers, Dordrecht (the Netherlands), 2001).

X. P. Xu and A. Needleman, Void nucleation by inclusions debonding in a crystal matrix, Modelling Simul. Mater. Sci. Eng. 1 (1993) 111-132.

Q. D. Yang, M. D. Thouless and S. M. Ward, Elastic-plastic mode-II fracture of adhesive joints, Int. J. Solids Struct. 38 (2001) 3251-3262.

F. Yoshida, M. Urabe, R. Hino and V. V. Toropov, Inverse approach to identification of material parameters of cyclic elasto-plasticity for component layers of a bimetallic sheet, Int. J. Plasticity 19 (2003) 2149-2170. 


\section{List of Tables}

1 Identified parameters as functions of the standard uncertainty level $s$ of the displacement data. . . . . . . . . . . . . 25

2 Values of the different identified parameters as a function of the $r$ ratio. . . . . . . . . . . . . . . . . . . . 31 


\section{List of Figures}

1 Joined sample (surface area: $297 \times 50 \mathrm{~mm}^{2}$ ) cut from the original laminate. The epoxy adhesive layer between the plate and the Z-shaped stringer is the focus of the present study.

2 Detail of the adhesive joint (in red), showing a tapered profile to allow for the insertion of the Z-shaped reinforcement on the skin laminate. Both laminates are made of three aluminum alloy (light gray) and two epoxy pre-preg (dark gray) layers. The small sub-domain monitored by DIC is located at the right end of the picture, where the joint thickness stabilizes to 60 $\mu \mathrm{m}$ (constant along the remaining length). The magnification used herein is less than that adopted in the pictures analyzed with DIC . . . . . . . . . . . . . . . . . . . .

3 Non-conventional test on a joined sample, up to complete debonding. . . . . . . . . . . . . . . . .

4 Sequence of digital images monitoring the debonding process of the adhesive layer (about $60 \mu \mathrm{m}$ thick) within the ROI after removal of rigid-body motions. The joint length shown in picture labeled 0 is $1.88 \mathrm{~mm}$. The (glossy) aluminum alloy layers fastened by the (dark) adhesive layer can be clearly distinguished, and the appearance of a completely traction-free surface is preceded by isolated thin fibrils bridging the opposite aluminum alloy surfaces, illuminated by light traversing the open microscopic crack. . . . . . . . . . . . . 8 
5 Measurements at the microscale provided by DIC through a finite element discretization. Deformed meshes of joined substrates are shown at given measurement instants $i$ during the debond test. .................... . . 9

$6 \quad$ Interface element with a finite thickness. . . . . . . . . . . . . 13

7 Profiles of normal tractions along the modeled interface, at different measurement instants $i$, computed with coarse (solid line) and fine (open circles) meshes. . . . . . . . . . . 16

8 Mechanical model (in plane stress) to simulate debonding within the monitored domain, in which boundary displacements estimated by DIC are prescribed. Deformed mesh at given measurement instants $i$ during the tests. . . . . . . . . . . . . . . 18

9 Sensitivity of the horizontal displacement field $u_{x}$ with respect to parameter $\delta_{t}, \partial u_{x} / \partial \delta_{t}$, at different instants $i$ during the test simulation. .................... . . 19

10 Validation of the identification procedure on the basis of artificial data. Minimization process of the objective function by Trust Region algorithm, in the presence of a Gaussian (noncorrelated) noise on measured displacements, with increasing standard deviation $s$, namely $s=0,0.1,0.6,1$ and 2 pixels. . 24

11 Change of normal and tangential traction profiles at the adhesive interface, $T_{n}$ and $T_{t}$, resp., re-constructed by the proposed identification procedure at different measurement instants $i$, with the a priori assumed ratio $r=4 \ldots \ldots . . . . . .27$ 
12 Interface tractions $T_{n}$ at measurement instants $i=8,11,14,17$, identified by the proposed inverse procedure for different val-

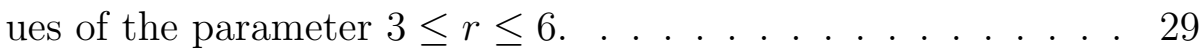

